\title{
8 Fazit: Lokale Relevanzsysteme bei der simultanen Koordination multipler Aktivitäten in Abhängigkeit von der strukturellen (In)Kompatibilität der Beteiligungsweisen
}

Gegenstand der vorliegenden Studie ist die gleichzeitige Relevanz multipler Aktivitäten in Interaktionen. Als Ergebnis der Analysen kann Folgendes festgehalten werden: Wenn in interaktionalen Kontexten für Beteiligte zwei oder mehr Aktivitäten gleichzeitig relevant werden, verwenden Teilnehmende entsprechende Koordinierungsverfahren, um die ko-relevanten Aktivitäten miteinander abzustimmen (8.1). Sind die multimodalen Beteiligungsweisen an den Aktivitäten strukturell kompatibel miteinander, können die Interagierenden Aktivitäten simultan vollziehen. Treten jedoch dauerhafte oder temporäre Inkompatibilitäten der Beteiligungsweisen auf, brechen Teilnehmende Aktivitäten ab bzw. pausieren diese, bis die benötigten multimodalen Ressourcen wieder zur Verfügung stehen (8.2). In den untersuchten Sequenzen aus Theaterproben handelt es sich bei den in den Hintergrund verschobenen Aktivitäten um solche, die jeweils weniger Relevanz für das aktuelle Projekt (z. B. eine Spielprobe im Rahmen der Erarbeitung eines Theaterstücks) aufweisen. Erfordert dabei ein solches Projekt den simultanen Vollzug ko-relevanter Aktivitäten mit jeweils strukturell inkompatiblen Beteiligungsweisen, greifen Beteiligte auf die Verfahren der Transformierung, Synchronisierung, Routinisierung und Priorisierung zurück. Diese Verfahren ermöglichen es, die strukturelle Inkompatibilität multipler Aktivitäten zumindest kurzzeitig lokal zu kompensieren (8.3). Auf diese Weise wird ein Blick in die Organisation lokaler Relevanzsysteme in Hinblick auf übergeordnete Projekte möglich (8.4).

\subsection{Gleichzeitige Relevanz multipler Aktivitäten als interaktionales Phänomen}

Gleichzeitigkeit in interaktionalen Kontexten kann konversationsanalytisch auf unterschiedlichen kommunikativen Ebenen verortet werden. Auf der sequentiellen Ebene finden sich beispielsweise Überlappungen (Jefferson 1986) als gleichzeitig relevante Sprachbeiträge oder schisming (Egbert 1993) bzw. side sequences (Jefferson 1972) als simultan nebeneinander existierende Interaktionsstränge. Der Ebene der multimodalen Simultanität ist das als Multimodalität (Mondada 2019a; Goodwin 2018) konzeptualisierte Nebeneinander endogener Ressour- 
cen zuzuordnen, auf die Interagierende beim Vollzug von Interaktionsbeiträgen zurückgreifen. Aus der sequentiellen Abfolge der multimodal organisierten Interaktionsbeiträge formt sich die Ebene der Aktivitäten, deren Phänomene der Simultanität in dieser Studie fokussiert werden. Die gleichzeitige Relevanz multipler Aktivitäten in Interaktionen kann unter anderem hierarchisch (main/ side involvements in activities, Goffman 1963), temporal (parallele Aktivitäten, Pitsch 2006) und koordinativ (multiactivity, Haddington et al. 2014) beschrieben werden. Die vorliegende Untersuchung folgt vor allem dem koordinativen Ansatz und betrachtet zunächst, mit welchen Koordinationsverfahren Interagierende auf eine gleichzeitige Relevanz multipler Aktivitäten reagieren. Als Aktivität wird dabei eine Sammlung aufeinander bezogener Beteiligungsweisen bezeichnet, die von den Teilnehmenden situativ als zusammengehörig behandelt werden (vgl. Kap. 2). Mithilfe multimodaler Beteiligungsweisen stellen die Interagierenden unter Verwendung situativer Praktiken (Schegloff 1997) die Teilnehmendenrahmung (participation framework, Goffman 1981; Goodwin \& Goodwin 2004; vgl. Levinson 1988) der jeweils aktuellen Situation her. Aktivitäten spannen damit einen Rahmen für die Handlungen auf, die sie umschließen (Levinson 2013; Goodwin 2000c). Mehrere Aktivitäten können für Interagierende dann relevant werden, wenn andere Mitglieder des aktuellen Interaktionsensembles (Schmitt 2012) neue Aktivitäten initiieren, die zu bereits bestehenden Aktivitäten hinzukommen - zum Beispiel wenn Teilnehmende einer laufenden Diskussion neu hinzukommende Beteiligte BEGRÜSSEN. Eine weitere Möglichkeit für das gleichzeitige Relevantwerden multipler Aktivitäten kann dann eintreten, wenn ein ${ }^{\star} \mathrm{e}$ Interagierende*r zusätzlich zu einer aktuell laufenden Aktivität eine neue Aktivität beginnt, die zunächst nur für ihn` ${ }^{\star}$ sie von Bedeutung ist - beispielsweise eine TRINK-Aktivität. Die Aktivitäten BEGRÜSSEN und TRINKEN unterscheiden sich in zwei wesentlichen Aspekten: der Teilnehmendenkonstellation und den Beteiligungsweisen. Die Teilnehmendenkonstellation einer Aktivität bestimmt darüber, inwiefern sich andere Teilnehmende des Interaktionsensembles an ihr beteiligen. Eine BEGRÜSSUNG wird typischerweise von mindestens zwei verschiedenen Interagierenden hergestellt, welche die Interaktionsbeiträge Gruß und Gegengru $\beta$ als Paarsequenz aufeinander bezogen vollziehen (Schegloff 1968; Sacks 1972). Eine solcherart aus den interaktionalen Beiträgen mehrerer Beteiligter hergestellte Aktivität kann als Ensembleaktivität bezeichnet werden. Trägt hingegen nur ein ${ }^{\star}$ Interagierende`r mit seiner`ihrer Beteiligung zu einer Aktivität bei, handelt es sich um eine Einzelaktivität wie beispielsweise dem TRINKEN. Bei Einzel- und Ensembleaktivitäten in Interaktionen handelt es sich um Kategorien der Partizipation. Dies ist bei Ensembleaktivitäten offensichtlich, wenn Teilnehmende ihre (auch verbal abstinenten, Heidtmann \& Föh 2007) Interaktionsbeiträge aufeinander ausrichten. Aber auch Einzelaktivitäten unterliegen - insofern sie Teil 
einer sozialen Situation (Goffman 1963) sind - einem Abstimmungsprozess mit den laufenden Vorgängen des Interaktionsensembles, der sich in der intra- und interpersonellen Koordinierung (Deppermann \& Schmitt 2007) ausdrückt. Neben der Teilnehmendenkonstellation unterscheiden sich Ensembleaktivitäten wie BEGRÜSSUNGEN und Einzelaktivitäten wie TRINKEN in ihren Beteiligungsweisen. Um an einer BEGRÜSSUNG teilzunehmen, verwenden Interagierende wenigstens verbale Beteiligungsweisen, etwa bei Telefongesprächen. In Situationen hingegen, in denen sich die Beteiligten von Angesicht zu Angesicht gegenüberstehen, können darüber hinaus noch weitere multimodale Beteiligungsweisen wie Winken (Licoppe 2017), Umarmungen (Goodwin 2017) und natürlich auch Blickkontakt (Rossano 2013) hinzukommen. Der Vollzug einer TRINK-Aktivität gestaltet sich demgegenüber vor allem mit haptischen Beteiligungsweisen, weshalb solche Einzelaktivitäten in der aktuellen Interaktionsforschung häufig auf die Beteiligung der Hände (Kamunen 2019) und/oder auf die Abwesenheit von Sprache (Deppermann \& Streeck 2018) reduziert werden. Im Rahmen dieser Studie hat sich jedoch gezeigt, dass auch Ensembleaktivitäten sprachfrei (z. B. TANZEN, vgl. Kap. 6) oder primär haptisch-proxemisch (z. B. ÜBERREICHEN DER AKTUELLEN STÜCKFASSUNG, vgl. Kap. 5) organisiert werden können, wodurch eine Kategorisierung der Aktivitäten nach Teilnehmendenkonstellation (Ensemble- oder Einzelaktivität) und nicht nach den verwendeten Beteiligungsweisen (verbal, gestisch, visuell, usw.) sinnvoll erscheint.

\subsection{Verfahren zur Bearbeitung struktureller Inkompatibilität multimodaler Beteiligungsweisen}

Treten mehrere Aktivitäten in einer sozialen Situation auf, an denen sich die Interagierenden beteiligen wollen, entstehen unterschiedliche Relevanzen auf den Ebenen der Teilnehmendenkonstellation und der Beteiligungsweisen, welche die Teilnehmenden koordinativ in Einklang bringen müssen. Dies kann dann der Fall sein, wenn eine Aktivität aufrechterhalten werden soll, während mit den multimodalen Beteiligungsweisen ein engagement display (Goodwin 1981) bezüglich der relevanten Aktivitäten angezeigt wird. Ein engagement display entsteht dadurch, dass mindestens eine Beteiligungsweise auf die jeweilige Aktivität ausgerichtet ist. Der öffentlich zugängliche Charakter einer somit erzeugten multimodalen Gestalt (Mondada 2014a) ermöglicht es Teilnehmenden, die laufende Aktivität als solche zu erkennen und auf sie interpersonell koordinativ in ihrem eigenen Verhalten zu reagieren. Wird jedoch eine Beteiligungsweise auch für eine andere Aktivität benötigt, liegt ein Relevanzproblem vor. Das tritt besonders bei einer strukturellen Inkompatibilität der Beteiligungsweisen gleichzeitig relevan- 
ter Aktivitäten zutage. So benötigen beispielsweise sowohl die BEGRÜSSUNG als auch das TRINKEN den Mund zur Realisierung der verbalen bzw. haptisch-proxemischen Beteiligungsweisen. Beide Aktivitäten verhalten sich demnach in ihren Beteiligungsweisen strukturell inkompatibel zueinander. Der Frage, wie Teilnehmende mit dieser koordinativen Anforderung umgehen, wurde im Zuge dieser Arbeit zuerst nachgegangen:

1. Wie gehen Interagierende in sozialen Situationen mit der Anforderung um, wenn gleichzeitig zwei (oder mehr) Aktivitäten relevant werden, die simultan dieselben multimodalen Beteiligungsweisen erfordern?

Wie die Analysen in Kapitel 5 gezeigt haben, können Teilnehmende diesem interaktionalen Problem mithilfe von drei Verfahren begegnen, die je nach struktureller (In)Kompatibilität der Beteiligungsweisen Anwendung finden können. Die drei Koordinierungsverfahren (serielle, quasi-simultane und simultane Koordinierung ko-relevanter Aktivitäten) unterscheiden sich jeweils im Grad der simultanen Realisierung (nicht-simultan, beinahe-simultan, simultan) der multiplen Aktivitäten und hinsichtlich der verwendeten Praktiken (Abbruch, Wiederaufnahme, Pausierung, Fortsetzung, Beteiligungsmarkierung).

Verhalten sich die Beteiligungsweisen von ko-relevanten Aktivitäten strukturell inkompatibel zueinander, können Beteiligte auf das Koordinationsverfahren der seriellen Koordinierung zurückgreifen. Hierbei stellen Teilnehmende eine Aktivität zugunsten einer anderen, gleichzeitig relevanten Aktivität durch einen Aktivitätsabbruch zurück. Abbrüche von Aktivitäten unterscheiden sich systematisch hinsichtlich ihrer Teilnehmendenkonstellation voneinander. Interaktionale Abbrüche von Einzelaktivitäten können ohne Account und ohne intersubjektive Gestaltung abgebrochen bzw. wiederaufgenommen werden. Im Gegensatz dazu ist es für die soziale Organisation der Ensembleaktivitäten von großer Bedeutung, ob die Abbrüche und Wiederaufnahmen accountable (Garfinkel 1967) gemacht werden. Einzelaktivitäten, die mithilfe von aktivitätsrelevanten Objekten (z. B. ein Smartphone oder eine Tasse) hergestellt werden, können dadurch abgebrochen werden, dass die Interagierenden ihre haptischen und visuellen Bindungen mit diesen Fokusobjekten auflösen. Im Unterschied dazu können Abbrüche von Ensembleaktivitäten mit verbalen Beteiligungsweisen erfolgen, indem die Interagierenden ihre verbalen und visuellen Orientierungen mit den Ko-Interagierenden der Ensembleaktivität aufheben und dadurch Nichtverfügbarkeit für die jeweilige Ensembleaktivität anzeigen. Der Abbruch ist für andere Interagierende damit als solcher erkennbar, wenn ein engagement display einer anderen (konkurrierenden) Aktivität relevant gesetzt wird. Die Relevanzsetzung einer Aktivität kann also dadurch entstehen, dass eine konkurrierende Aktivität von den 
Beteiligten in den Hintergrund (Langacker 2008) gerückt wird. Eine solche Relevanzverschiebung innerhalb einer sozialen Situation zeigt sich dann, wenn Teilnehmende die Bearbeitung bzw. Ausrichtung auf eine der laufenden Aktivitäten einstellen. Diese Abbrüche unterscheiden sich je nach den Beteiligungsweisen, mit denen die Beteiligten die Aktivitäten hervorbringen. Aktivitäten, die haptische Beteiligungsweisen (z. B. das Anfassen eines Objektes) erfordern, werden durch die Teilnehmenden abbrechend zurückgestellt, indem eben diese Beteiligungsweisen aufgegeben werden. Intersubjektiv organisierte Aktivitäten werden von den Interagierenden dadurch abgebrochen, dass die zur Herstellung von Intersubjektivität verwendeten Beteiligungsweisen (z. B. verbale oder visuell-proxemische Beteiligungsweisen) aufgelöst werden. Die Folge von Aktivitätsabbrüchen und -wiederaufnahmen ist eine Aktivitätsserie, die dadurch gekennzeichnet ist, dass Teilnehmende immer nur ein engagement display bzgl. einer der gleichzeitig relevanten Aktivitäten anzeigen. Die gleichzeitige Relevanz wird somit in eine serielle Abfolge der Aktivitäten umorganisiert. Aktivitätsabbrüche aufgrund von struktureller Inkompatibilität können dann auftreten, wenn multiple Aktivitäten unterschiedliche Aufmerksamkeitsfelder (z. B. visuelle Orientierung eines ${ }^{\star}$ r Teilnehmenden auf eine ${ }^{\star} n$ Ko-Interagierende ${ }^{\star} n$ vs. auf ein nur für ihn ${ }^{\star}$ sie zugängliches semiotisches Feld) und/oder physische Aufwendung (z. B. händische Bearbeitung von Objekten) erfordern. Die Analysen haben allerdings darüber hinaus gezeigt, dass Aktivitätsabbrüche keineswegs nur die Reaktion der Beteiligten auf eine eventuelle kognitive und/oder körperliche Mehrbelastung sind. Vielmehr spielt die strukturelle Integrierbarkeit der notwendigen Beteiligungsweisen innerhalb der aktuellen sozialen Situation eine übergeordnete Rolle. So ist für denselben Interagierenden einerseits möglich, die Einzelaktivitäten TRINKEN und AUF DEM SMARTPHONE IM NEWSFEED LESEN gleichzeitig mit visuellen und haptischen Beteiligungsweisen zu vollziehen (Kap. 5, Transkript 16), während er andererseits seine SMARTPHONE-Einzelaktivität abbricht, als im Rahmen der Ensembleaktivität BEGRÜSSUNG haptische (Händeschütteln), visuelle (Blickkontakt) und verbale Beteiligungsweisen (Gegengruß) durch die Interaktion mit einem Ko-Akteur relevant werden (Kap. 5, Transkript 1). In anderen Fällen (Kap. 5, Transkripte 2, 3 und 5) werden Ensembleaktivitäten zugunsten von Einzelaktivitäten abgebrochen. Darin zeigt sich, dass die Teilnehmenden den Ensembleaktivitäten nicht per se höhere Wichtigkeit einräumen als den Einzelaktivitäten, sondern in ihrem Abbruch lediglich anzeigen, welcher der multiplen Aktivitäten sie innerhalb der aktuellen sozialen Situation mehr Relevanz zuschreiben.

Ein solches lokales Relevanzsystem der multiplen Aktivitäten kommt auch dann zum Ausdruck, wenn Interagierende dem interaktionalen Problem der Gleichzeitigkeit dadurch begegnen, dass sie sich an den für sie simultan relevanten Aktivitäten zur gleichen Zeit beteiligen. Dies kann entweder als tatsächliche 
simultane Bearbeitung (siehe unten) oder als quasi-simultane Koordination erfolgen. Besonders bei der letzteren Form wird mittels der Fortsetzungsprojektion eine Praktik erkennbar, mit der Teilnehmende ihre lokale Relevanz der multiplen Aktivitäten ordnen. Mithilfe von Pausierungen und Fortsetzungen werden ko-relevante Aktivitäten eines Interaktionsensembles so koordiniert, dass die gleichzeitige Relevanz der Aktivitäten aufrechterhalten bleiben kann - jedoch nur eine Aktivität bearbeitet wird. Für die andere(n), nicht bearbeitete(n) Aktivität(en) wird ein engagement display hergestellt, in dem eine Fortsetzungsprojektion angelegt ist. Diese kann den Ko-Interagierenden anzeigen, dass die pausierte Aktivität relevant gehalten wird und von einer zeitnahen (Wieder-)Bearbeitung ebendieser Aktivität ausgegangen werden kann. Hebt der die Interagierende die Fortsetzungsprojektion auf, ohne sie einzulösen, erlischt die Beteiligung an der jeweiligen Aktivität und sie wird in einen Aktivitätsabbruch überführt, wodurch die lokale Relevanz der vormals pausierten Aktivität weiter in den Hintergrund verschoben wird. Fortsetzungsprojektionen von Einzelaktivitäten mit zentralen haptischen Beteiligungsweisen können beispielsweise hergestellt werden, indem diese Beteiligungsweisen pausiert werden, aber mindestens ein Aspekt darauf hinweist, dass die Aktivität (jederzeit) wiederaufgenommen werden kann. Beispiele dafür sind etwa das Offenhalten einer Taschenlasche beim AusPACKEN (Kap. 5, Transkript 6) oder Aufbehalten eines Rucksacks, während weiterhin die Hände die Riemen im Rahmen einer KLEIDUNG-ABLEGEN-Einzelaktivität umfasst halten (Kap. 5, Transkript 7). Analog dazu können Fortsetzungsprojektionen bei Ensembleaktivitäten mit pausierten verbalen Beteiligungsweisen hergestellt werden, indem syntaktisch oder thematisch projiziert wird, dass ein Turn weitergeführt werden wird (Kap. 5, Transkript 8). Fortsetzungsprojektionen stellen eine konstante Herstellungsleistungen der Beteiligten dar, mithilfe derer sie pausierte Aktivitäten relevant halten, während sie gleichzeitig eine konkurrierende Aktivität bearbeiten.

Voraussetzung dafür, dass eine Aktivität bei Ko-Relevanz mit mindestens einer weiteren Aktivität pausiert und nicht abgebrochen wird, ist eine zumindest teilweise strukturelle Kompatibilität. Das bedeutet, dass die ko-relevanten Aktivitäten im Kern nicht auf dieselben Beteiligungsweisen zurückgreifen, was im Gegensatz dazu bei der durch strukturelle Inkompatibilität geprägten seriellen Koordinierung der Fall ist. Dennoch ist es möglich, dass eine Beteiligungsweise einer Aktivität den Vollzug einer anderen Beteiligungsweise zwar nicht ausschließt, aber zumindest verhindert. Dies zeigt sich etwa, wenn die visuelle Beteiligungsweise an einem VoRSCHLAG dazu genutzt wird, um Blickkontakt mit der Interaktionspartnerin zu halten, dies jedoch das Lokalisieren eines Ablageplatzes eines AUSGEPACKTEN Objekts verhindert. Diese Konstellation hat das Pausieren des AusPaCKEns zur Folge, bis die visuelle Beteiligungsweise zum Lokalisieren 
frei wird oder der Teilnehmer auf eine verbale Rückmeldepartikel zur Beteiligungsmarkierung bei abgebrochenem Blickkontakt zurückgreift (Kap. 5, Transkript 13). Ein essentieller Aspekt von Pausierung ist somit der Umstand, dass mindestens eine Beteiligungsweise auf die jeweilige Aktivität verweist, zu deren Vollzug sie eingesetzt wird, ohne die Aktivität aktiv zu bearbeiten. Die Pausierung dauert an, bis die Beteiligungsweise wieder zur aktiven Bearbeitung eingesetzt werden kann oder abgebrochen werden muss. Fortsetzungen pausierter Aktivitäten erfolgen in den besprochenen Sequenzen an solchen Stellen, an denen sich die gleichzeitige Relevanz, die eine Pausierung zuvor notwendig gemacht hat, aufgelöst hat, z. B. wenn eine verbale Beteiligung an einer Ensembleaktivität verweigert (Kap. 5, Transkript 9), die konditionelle Relevanz innerhalb einer Ensembleaktivität eingelöst (Kap. 5, Transkript 10) oder eine Ensembleaktivität gemeinsam abgeschlossen wird (Kap. 5, Transkript 11). Sowohl Wiederaufnahmen abgebrochener Aktivitäten als auch Aktivitätsfortsetzungen zeigen an, dass die Relevanz der betreffenden Aktivität für die Teilnehmenden im Hintergrund fortbestanden hat, jedoch bis zur Fortsetzung nicht aktiv bearbeitet wurde. Wiederaufnahmen und Fortsetzungen von Aktivitäten, die im Rahmen gleichzeitiger Relevanz zurückgestellt wurden, unterscheiden sich hinsichtlich des Grades, zu dem die jeweilige Aktivität in den Hintergrund verschoben wurde, was sich im engagement display anhand der Fortsetzungsprojektion und der intersubjektiven Gestaltung zeigt. Wiederaufgenommene Ensembleaktivitäten werden von den Teilnehmenden in den analysierten Sequenzen (z. B. Kap. 5, Transkript 5) deutlich expliziter als solche gerahmt als Fortsetzungen (z. B. Kap. 5, Transkript 8). Wiederaufnahmen und Fortsetzungen sind demnach Praktiken der seriellen und quasi-simultanen Koordination multipler Aktivitäten, bei denen die Relevanz der Aktivitäten im Hintergrund in unterschiedlichem Maße fortbesteht, obwohl keine aktive Bearbeitung zu beobachten ist.

Die dritte Möglichkeit, mit der Interagierende auf die gleichzeitige Relevanz multipler Aktivitäten reagieren können, ist die simultane Koordinierung der zeitgleich auftretenden Aktivitäten einer sozialen Situation. Essentiell dafür ist die strukturelle Kompatibilität der multimodalen Beteiligungsweisen der ko-relevanten Aktivitäten. So können Teilnehmende etwa per Blickkontakt und Nicken Beteiligung an ERKLÄR-Ensembleaktivität anzeigen, während sie gleichzeitig mithilfe haptischer Beteiligungsweisen eine AusPACK-Einzelaktivität vollziehen (Kap. 5, Transkript 12). Wenn Einzelaktivitäten visuelle Beteiligungsweisen erfordern und daraufhin der Blickkontakt zwischen den Interagierenden aufgelöst wird, kann ein engagement display bzgl. einer Ensembleaktivität auch per Rückmeldepartikel erfolgen (Kap. 5, Transkripte 13 \& 14). Wie die Analysen gezeigt haben, erfordern simultan koordinierte Aktivitäten neben einer steten interpersonellen Koordinierungsleistung vor allem auch eine intrapersonelle Koordinie- 
rung. Das kann etwa beinhalten, dass Interagierende sich mit einer Hand gestikulatorisch an einer Aktivität beteiligen, während sie zeitgleich mit der anderen Hand die haptische Beteiligungsweise an einer anderen Aktivität realisieren (Kap. 5, Transkript 15). Eine simultane Bearbeitung multipler Aktivitäten stellt allerdings nicht nur koordinative Anforderungen an die Beteiligten. Stattdessen kann eine Simultanbearbeitung den Teilnehmenden ermöglichen, unterschiedlichen interaktionalen Zielen nachzugehen (z. B. etwas zu TRINKEN und gleichzeitig eine NACHRICHT AUF DEM SMARTPHONE ZU LESEN, Kap. 5, Transkript 16) oder komplexe gemeinsame Projekte zu realisieren (z. B. die ÜBERGABE eines Dokuments, dessen Inhalt gleichzeitig ERKLÄRT wird, Kap. 5, Transkript 17). Gemeinsame Projekte kennzeichnet eine zumindest teilweise routinisierte Ausrichtung auf ein antizipierbares interaktionales Ziel, das bestimmte Handlungsverläufe und Akteure erwartbar macht (Luckmann 1995).

\subsection{Verfahren zum simultanen Vollzug ko-relevanter Aktivitäten bei struktureller (In)Kompatibilität der multimodalen Beteiligungsweisen}

Wie die Analysen in Kapitel 6 und 7 gezeigt haben, unterstützt eine routinisierte Ausrichtung innerhalb von gemeinsamen Projekten den simultanen Vollzug korelevanter Aktivitäten. Unklar war dabei im Rahmen der multiactivity-Forschung bislang, mithilfe welcher Verfahren Interagierende - auch strukturell inkompatible - Teil-Aktivitäten simultan koordinieren, um im Rahmen der so entstehenden Multiaktivitäten komplexe Projekte bearbeiten zu können:

\section{Unter welchen Bedingungen ist ein simultaner Vollzug multipler Aktivitäten trotz} struktureller Inkompatibilität der multimodalen Beteiligungsweisen möglich?

In dieser Studie wurde untersucht, wie Beteiligte die gemeinsamen Projekte Tanzerarbeitung (Kap. 6) und Spielprobe (Kap. 7) mit strukturell kompatiblen EnsembleMultiaktivitäten (Kap. 6). bzw. strukturell inkompatiblen Einzel-Multiaktivitäten (Kap. 7) herstellen. Die Analysen zeigen, dass die Teilnehmenden - trotz Unterschieden bezüglich der projektspezifischen Praktiken - die gleichen Verfahren verwenden, um die Projekte (auch bei struktureller Inkompatibilität der korelevanten Aktivitäten) simultan zu koordinieren: Transformierung, Synchronisierung, Routinisierung und Priorisierung. Wie die Analysen zeigen konnten, greifen Interagierende bei der simultanen Bearbeitung von Multiaktivitäten im Rahmen eines gemeinsamen Projektes auf diese Verfahren zurück, die ein lokales Relevanzsystem ausprägen. Dieses liegt auch simultan koordinierten strukturell kom- 
patiblen Multiaktivitäten zugrunde, tritt aber insbesondere dann deutlich zum Vorschein, wenn strukturell inkompatible ko-relevante Aktivitäten eine simultane Koordinierung zum Aufrechterhalten eines Projekts erfordern. Denn wie in Kapitel 5 gezeigt werden konnte, greifen Interagierende bei strukturell inkompatiblen ko-relevanten Aktivitäten bei verschiedenen Projekten auf Abbruchspraktiken im Rahmen des seriellen Koordinationsverfahrens zurück. Demnach kommt dem Umstand eine besondere Bedeutung zu, dass die gleichzeitig relevanten Aktivitäten des gemeinsamen Projekts Spielprobe in den Daten trotz struktureller Inkompatibilität von den Beteiligten nicht abgebrochen oder pausiert, sondern simultan koordiniert werden und dies im lokalen Relevanzsystem erkennbar wird.

Mithilfe von Praktiken der Transformierung können Teilnehmende die Multiaktivitäten je nach kommunikativem Bedarf des aktuellen Projekts herstellen oder auflösen (Kap. 6). So werden Multiaktivitäten, die aus simultan koordinierten Ensembleaktivitäten bestehen (Ensemble-Multiaktivitäten), dadurch möglich, dass Beteiligte eine bestehende Monoaktivität um eine neu hinzukommende Aktivität zu einer Multiaktivität erweitern. Dies ermöglicht es ihnen, komplexe Interaktionsaufgaben zu bearbeiten, im Rahmen derer sie etwas beschreiben, was sie zeitgleich ausführen, etwa bei einem TANZENDEN-Vorschlag (Kap. 6, Transkript 18), einer TANZENDEN-Zusammenfassung (Kap. 6, Transkript 19) oder einer TANZENDEN-BitTE (Kap. 6, Transkript 20). Um solche Ensemble-Multiaktivitäten herzustellen, gleichen die Interagierenden in der Startphase der hinzukommenden Aktivität diese so an die bestehende Aktivität an, dass beide Teil-Aktivitäten simultan gestartet werden können. In den drei aufgeführten Fällen sind die Teil-Aktivitäten jeweils auf das gemeinsame Projekt Tanzerarbeitung ausgerichtet und dafür gleichermaßen relevant. Verändert sich nun die Relevanz einer der Teil-Aktivitäten (z. B., wenn die Beteiligung einer weiteren Person eingefordert wird, Kap. 6, Transkript 21), kann diese Teil-Aktivität so verändert werden, dass sie der situativen Anforderung gerecht wird. Auf diese Weise muss die Multiaktivität nicht gänzlich aufgelöst werden, sondern bedarf lediglich eines Übergangs von einer Aktivität zu einer anderen Aktivität als neue Teil-Aktivität der laufenden Multiaktivität. So kann je nach situiertem Erfordernis aus einer TANZENDEN-ANWEISUNG eine TANZENDE-ERKLÄRUNG werden, die wieder zurück in eine TANZENDE-ANWEISUNG überführt wird, sobald die Teilnehmenden der Teil-Aktivität ERKLÄRUNG keine unmittelbare Relevanz mehr zuschreiben (Kap. 6, Transkript 22). Ist die lokale Relevanz einer der Teil-Aktivitäten einer Multiaktivität erloschen und tritt keine neue Aktivität an ihre Stelle, kann die Komplexität einer Multiaktivität verringert werden, indem nicht mehr relevante Teil-Aktivitäten aufgelöst werden. Die verbleibenden Aktivitäten können daraufhin als Monoaktivität fortgeführt werden. So wird aus der Ensemble-Multiaktivität TANZENDE-RÜCKVERSICHERUNG die Ensemble-Monoaktivität RÜCKVERSICHERUNG, sobald die TANZ-Aktivität im Rahmen der 
Situation für die Bearbeitung des aktuellen Projekts nicht mehr benötigt wird (Kap. 6, Transkript 30). Analog dazu wird aus einer TANZENDEN-ANWEISUNG durch eine solche Gestaltreduzierung die Ensembleaktivität ANWEISUNG, sobald für die Teilnehmenden eine Veränderung im Aktivitäts- bzw. Handlungsrahmen (action frame transition, Reed 2015) erkennbar ist und die TANZ-Ensembleaktivität situativ an lokaler Relevanz verliert (Kap. 6, Transkript 31). Ändert sich der Aktivitätsrahmen innerhalb des gemeinsamen Projekts (z. B. wenn im Rahmen einer Tanzerarbeitung das eben Erarbeitete nun selbstständig wiederholt werden soll, Kap. 6, Transkript 32), lösen die Beteiligten die Gestalt der laufenden Multiaktivität gemeinsam auf. Bei einer solchen Gestaltauflösung erlischt die Relevanz aller Teil-Aktivitäten einer Multiaktivität. Deren Gestalt wird schrittweise reduziert, bis alle Teil-Aktivitäten beendet sind und die multimodalen Ressourcen der Teilnehmenden für Folgeaktivitäten neu verteilt werden können. Beispielsweise kann so im Rahmen der Multiaktivität TANZENDER-VoRSCHLAG zunächst die Teil-Aktivität TANZEN und dann die verbleibende Teil-Aktivität VoRsCHLAGEN abgeschlossen werden, um anschließend mit einer SzENISCHEN PROBE als neuer, für das Projekt Tanzerarbeitung relevanter Aktivität zu beginnen (Kap. 6, Transkript 33). Daran zeigt sich, dass Interagierende Multiaktivitäten im Rahmen des Verfahrens einer Transformation in situ je nach kommunikativem Bedarf für die Bearbeitung des Projekts herstellen oder auflösen. Das somit hervortretende lokale Relevanzsystem der multiplen Aktivitäten macht dabei deutlich, welche aktuelle Bedeutung die Interagierenden den einzelnen Aktivitäten im Rahmen des übergeordneten Projekts (bzw. dem gemeinsamen Projekt selbst) zuschreiben.

Um Multiaktivitäten aufrechtzuerhalten, können Interagierende auf das Verfahren der Synchronisierung zurückgreifen. Dabei gleichen sie ihre Beteiligungsweisen an den Teil-Aktivitäten der Multiaktivitäten intrakoordinativ miteinander (Selbstsynchronisierung, Kap. 6, Transkripte 23 \& 27) und interkoordinativ mit den Ko-Teilnehmenden des Interaktionsensembles (interaktionale Synchronisierung, Kap. 6, Transkripte 25 \& 26) in Bezug auf Form, Intensität und Geschwindigkeit der verwendeten, strukturell kompatiblen Beteiligungsweisen ab bzw. an. Die Synchronisierung im Rahmen der Multiaktivität hat den funktionalen Mehrwert, dass Interagierende dadurch in der Lage sind, mehrere kommunikative Funktionen eines gemeinsamen Projekts gleichzeitig zu leisten. So kann beispielsweise die TANZ-Teilaktivität einer TANZENDEN-ANWEISUNG andere Informationen für die Tanzerarbeitung liefern (unter anderem Tanzhaltung oder Tanzrichtung) als die Teil-Aktivität ANwEIsung (unter anderem Taktvorgabe oder Ankündigung einer nächsten Tanzfigur, die umgesetzt werden soll) (Kap. 6, Transkript 24). Das Zusammenspiel der Teil-Aktivitäten als syntactic-bodily gestalt (Keevallik 2015) ermöglicht es den Interagierenden in den analysierten Fällen, das komplexe Projekt so zu bearbeiten, dass sie die Ziel-Aktivität TANZEN der 
Tanzerarbeitung bereits ausführen, während sie diese noch erarbeiten. Darüber hinaus können Interagierende im Rahmen synchronisierter Multiaktivitäten Aspekte einer der Teil-Aktivitäten hervorheben, während weitere Teil-Aktivitäten ohne explizite Rahmung fortgeführt werden und damit deren gleichzeitige Relevanz aufrechterhalten bleibt. Dies ist etwa der Fall, als die Choreographin im Rahmen einer TANZENDEN-ANWEISUNG (Kap. 6, Transkript 28) mithilfe aspektueller Hervorhebung als Synchronisierungspraktik die zu erarbeitende Tanzchoreographie sukzessive aufbaut, während sie diese vorführt. Die beiden gleichzeitig mittanzenden Angewiesenen können dadurch die markierten Aspekte des Tanzes beibehalten und additiv neue Aspekte mit der TANz-Gestalt synchronisieren, die in der Folge schrittweise an Komplexität zunimmt. Dabei beziehen sich beide Teil-Aktivitäten auf dasselbe Projekt (Tanzerarbeitung), das den interaktionalen Rahmen für die multiplen Aktivitäten definiert. Es scheint daher für die Interagierenden nicht notwendig zu sein, dass sich synchronisierte Teil-Aktivitäten funktional doppeln. Stattdessen erlaubt die Rahmung des gemeinsamen Projekts, auf das sich die Beteiligten ausrichten, die Fokussierung auf einzelne Aspekte einer der Teil-Aktivitäten. Gleichzeitig behandeln die Beteiligten weitere synchronisierte Teil-Aktivitäten der Multiaktivität als nach wie vor relevant, indem sie ihre auf diese ausgerichteten Beteiligungsweisen störungsfrei fortsetzen. Das liefert einen wichtigen Hinweis darauf, wie Multiaktivitäten aufrechterhalten werden können und welche Rolle dabei das übergeordnete Projekt spielt: Das gemeinsame Projekt spannt den kontextuellen Rahmen auf, innerhalb dessen Interagierende simultan koordinierte multiple Aktivitäten als Teil-Aktivitäten der Multiaktivitäten per Synchronisierung miteinander verbinden können.

Zur gleichzeitigen Bearbeitung simultan relevanter multipler Aktivitäten kön nen Interagierende darüber hinaus auf das Verfahren der Routinisierung zurückgreifen. Durch mehr oder weniger festgelegte Abläufe sind Beteiligte dabei in der Lage, bestimmte Bewegungstrajektorien vorauszuahnen und sich prospektiv darauf auszurichten (vgl. Streeck \& Jordan 2009). Auf diese Weise ist es den Interagierenden möglich, auf Veränderung im Rahmen von synchronisierten Aktivitäten zu reagieren, ohne die Synchronisierung selbst aufzulösen. Dies ist zum Beispiel der Fall, als der Schauspieler einen Zwischenschritt seiner Tanzpartnerin ausgleicht, indem er die Fußposition zum nächsten Taktschlag antizipieren und darauf mit einem verlängerten Schritt seinerseits reagieren kann (Kap. 6, Transkript 25). Ein anderes Beispiel findet sich in der Sequenz, in der die Schauspielerin anhand ihres Tanzpartners eine TANZENDE-BITTE realisiert und er sich anhand interkorporaler Koordination an dieser Multiaktivität beteiligen kann (Kap. 6, Transkript 20). Die künstlerischen Projekte Tanzerarbeitung (Kap. 6) und Spielprobe (Kap. 7) bieten in ihren verabredeten Abläufen Strukturen an, in denen Interagierende die Erwartbarkeit von routinisierten Abläufen zur Koordination 
nutzen können. So ist die Regieassistentin anhand des im Textbuch festgelegten Geschehens in der Lange zu antizipieren, welche*r der Spielenden als nächstes sprechen wird und potentiell einen Hänger haben könnte. Das ermöglicht ihr ihre visuelle Aufmerksamkeit auf die entsprechende Person auszurichten, um gegebenenfalls sofort eingreifen zu können, wenn der Fortschritt des Projekts Spielprobe in Gefahr gerät (Kap. 7, Transkript 37). In einem anderen Fall ermöglicht ihr der zu gewissen Teilen erwartbare Ablauf der Aktivität SZENISCHEs SPIEL einen sequentiellen Slot zu erkennen, in dem sie eine ebenfalls relevante Aktivität (Mitschreiben) vollziehen kann, ohne die bestehende Multiaktivität abzubrechen oder pausieren zu müssen (Kap. 7, Transkript 39). Dass die Aktivitäten in solchen Projekten trotz verabredeter Choreographie und Textbuch keinen vordefinierten Handlungsplänen im Sinne von ,ppre-scripted routines“ (Robinson 2013: 264) folgen, sondern das Resultat koordinativer Prozesse sind, zeigt sich deutlich anhand der Koordinationsweisen. Diese sind so gestaltet, dass auch unerwartete, spontan relevant werdende Aktivitäten wie eine ANFRAGE des Regisseurs an die Regieassistentin (Kap. 7, Transkript 43) in diese Multiaktivitäten integriert werden können. Am Rand der Theaterproben (Kap. 5) hingegen, also in Situationen, in denen Interagierende ihre Aktivitäten zwar auf das gemeinsame Projekt Erarbeitung eines Theaterstücks ausrichten (z. B. indem sie eine neue Inszenierungsidee vorschlagen, Kap. 5, Transkripte 6-8), diese Aktivitäten jedoch analog zu Alltagsaktivitäten (z. B. ERzÄHLEN oder BERICHTEN) vorwiegend in Bezug auf die verbalen Beteiligungsweisen einem erwartbaren Muster folgen (z. B. als kommunikative Gattung, Günthner \& Knoblauch 1994; verfestigte prosodische Gestalten und grammatische Konstruktionen, Günthner 2018, oder im Rahmen einer konditionellen Relevanz innerhalb einer Paarsequenz, Schegloff 1968) und keinen reproduzierbaren Ablauf zum Ziel haben, ist die Verwendung von Routinisierung als Koordinationsverfahren von Aktivitäten mit weniger starkem Bewegungsanteil für die Beteiligten von geringerer Relevanz. Das Antizipieren und Projizieren von routinisierten Abläufen kann Interagierenden in Interaktionsensembles also helfen, multiple Aktivitäten simultan zu bearbeiten - besonders bei struktureller Inkompatibilität der ko-relevanten Aktivitäten -, bleibt aber (soweit aus den vorliegenden Daten ersichtlich) größtenteils auf solche Anwendungsbereiche reduziert, in denen die Teil-Aktivitäten einer Multiaktivität erwartbaren Strukturen folgen (wie einem Tanz oder einer Theaterszene).

Teilnehmende schreiben simultan koordinierten Aktivitäten nicht zwangsläufig die gleiche Relevanz für das aktuelle Projekt zu. Solche Unterschiede in der Relevanz drücken sich in den situativen Verfahren der Priorisierung aus. Die Priorisierung zeigt sich lokal, wenn sich die Aktivitäten der Teilnehmenden strukturell inkompatibel zueinander verhalten und sich Beteiligungsweisen teilen. Das hat zur Folge, dass sich die Interagierenden entscheiden müssen, 
für welche der Aktivitäten sie diese Beteiligungsweisen verwenden. Wenn beide Aktivitäten für das gemeinsame Projekt gleich (un)wichtig sind, können Interagierende die betreffenden Beteiligungsweisen alternieren (Kap. 5, Transkript 16). Ist jedoch eine Aktivität stärker in das aktuelle Projekt eingebunden als eine andere, wird die relevantere Aktivität von den Beteiligten priorisiert. Solche Priorisierungen sind etwa zu beobachten, wenn die Regieassistentin im Rahmen von SoUfFLAGE-Multiaktivitäten die parallel zur Ensembleaktivität SZENISCHES SPIEL relevanten Einzelaktivitäten MitLesen und SCHREIBEN zu bearbeiten hat, die allesamt eine visuelle Beteiligungsweise verlangen (Kap. 7, Transkripte 37-40). Der Abzug der visuellen Beteiligung von der ScHREIB-Aktivität zugunsten der MitLESE- oder SPIEL-Aktivität zeigt an, dass die Regieassistentin in diesen Situationen der ScHREIB-Aktivität weniger lokale Relevanz zuschreibt. Geringere lokale Relevanz bedeutet dabei jedoch keinesfalls, dass diese Aktivität irrelevant oder optional wäre. Vielmehr haben die Analysen in Kapitel 7 gezeigt, dass die Beteiligten mithilfe der verschiedenen Aktivitäten unterschiedlichen Funktionen für das gemeinsame Projekt nachgehen. Der strukturellen Inkompatibilität von ko-relevanten Aktivitäten, die aufgrund ihrer Funktion für das gemeinsame Projekt eine simultane Koordinierung erfordern, können Teilnehmende mit Kompensationspraktiken begegnen. Hierbei handelt es sich um Praktiken, auf die Interagierende zurückgreifen können, um bei struktureller Inkompatibilität von Beteiligungsweisen keine der ko-relevanten Aktivitäten abbrechen oder pausieren zu müssen. Kompensationspraktiken gleichen zeitweise das Fehlen einer Beteiligungsweise aus, sodass die Aktivität trotzdem vollzogen werden kann. Indem Interagierende die weniger priorisierten Aktivitäten so realisieren, dass sie entweder durch teil-autonome (z. B. „,blindes“ MitschreiBen) oder antizipierende Organisation (z. B. beim „speichernden“ MITLESEN) auch dann vollzogen werden können, wenn die eigentlich benötigten Beteiligungsweisen für die priorisierten Aktivitäten verwendet werden. Dadurch zeigt sich bei der Bearbeitung simultaner Aktivitäten zum einen eine Präferenz der Teilnehmenden von solchen Aktivitäten, die relevanter für das aktuelle Projekt sind und zum anderen eine Gestaltung der Aktivitäten, die trotz struktureller Inkompatibilität der Beteiligungsweisen eine simultane Koordinierung ermöglichen. Wie sich hinsichtlich der Priorisierung ebenfalls gezeigt hat, priorisieren Interagierende solche Aktivitäten, die lokal relevant sind zugunsten von Aktivitäten, die sich vorwiegend global auf ein Projekt beziehen. Ein solches Relevanzsystem illustriert der Fall (Kap. 6, Transkript 29), in dem die Choreographin eine Tanzfigur zunächst global für das Projekt Tanzerarbeitung rechtfertigt, dann aber in eine lokal relevante ANwEISUNG transformiert, als sich die Synchronisierung der bestehenden Multiaktivität aufzulösen beginnt. 


\subsection{Coda: Lokale Relevanzsysteme bei der Bearbeitung struktureller (In)Kompatibilität multimodaler Beteiligungsweisen multipler Aktivitäten}

Anhand der Verfahren, mit denen Interagierende das gleichzeitige Auftreten multipler Aktivitäten bearbeiten, zeigen sie also ihr Verständnis der lokalen Relevanzen innerhalb der aktuellen sozialen Situation an. Dabei stimmen sie die lokale Relevanz, die sich auf die situativen Anforderungen bezieht, mit der globalen Relevanz ab, die einzelne (Multi)Aktivitäten für das übergeordnete Projekt haben können. Die vorliegende Studie macht auf diese Weise deutlich, dass die Goffman'schen (1963) Kategorien der main bzw. side activites nicht nur zu statisch gedacht (vgl. Mondada 2014c: 70), sondern vor allem auch zu grob gefasst sind, um die koordinative Komplexität von multiplen Aktivitäten adäquat beschreiben zu können. Denn wie besonders die Analysen zur SouffLAGE in Kapitel 7 gezeigt haben, lassen sich die vollzogenen Aktivitäten nicht trennscharf in Haupt- oder Nebenaktivitäten unterscheiden. Stattdessen kann es je nach situativer Ausgestaltung mehrere Aktivitäten geben, denen Beteiligte je nach Funktion für das überspannende Projekt unterschiedliche lokale Relevanzen zuschreiben. Auch käme mit dieser Kategorisierung die Frage auf, für welche Personen des Interaktionsensembles eine Aktivität einen Haupt- oder Nebenstatus hat. In den Abbrüchen oder Pausierungen von Ensembleaktivitäten zugunsten von Einzelaktivitäten zeigt sich, dass gemeinsame Aktivitäten mit sprachlicher Beteiligung nicht automatisch als Hauptaktivitäten bezeichnet werden können. Im Gegensatz dazu kann im Rahmen der SoufFLAGE eine LESE-Einzelaktivität unmittelbare Relevanz für den Fortschritt der laufenden Ensembleaktivität haben (was sie für Goffman zur Hauptaktivität machen würde), obwohl sich nur eine Person an dieser Aktivität beteiligt, was bei Goffman ein Hinweis für eine Nebenaktivität wäre. Fragt man also nach den Funktionen von simultaner Koordination multipler Aktivitäten, greift die Goffman'sche Kategorisierung zu kurz, weshalb Aktivitäten in der vorliegenden Arbeit über die Beteiligung kategorisiert wurden. Wie im Zuge der vorliegenden Studie ebenfalls deutlich wurde, nutzen Interagierende für die Koordination multipler Aktivitäten solche Verfahren, die sie auch für die interaktionale Herstellung von Monoaktivitäten verwenden, z. B. die delay-Organisation (Deppermann \& Schmitt 2007) oder die Synchronisierung (Pfänder, Herlinghaus \& Scheidt 2017). Zum ersten Mal wurde in dieser Studie im Kontext multipler Aktivitätsrelevanz gezeigt, dass diese Verfahren bei der Koordination multipler Aktivitäten nicht auf einzelne Ressourcen, sondern holistisch auf die jeweiligen Aktivitäten angewendet werden. Das lässt sich damit begründen, dass Interagierende in Situationen mit gleichzeitiger Relevanz von Aktivitäten nicht nur ihre multimodalen Ressourcen, sondern mitunter ganze Ressourcenbündel in Form 
von Beteiligungsweisen für mehrere ko-relevante Handlungsverläufe organisieren müssen. Die interaktionalen Implikationen davon zeigen sich immer dann, wenn aufgrund von nichtverfügbaren oder strukturell inkompatiblen multimodalen Ressourcen Beteiligungsweisen nicht ausgeführt werden können und die damit verbundenen Aktivitäten pausiert oder abgebrochen werden müssen. Das kann sich insofern auf die Interaktionen auswirken, als sich aufgrund solcher serieller oder quasi-simultaner Koordinationspraktiken die Teilnehmendenrahmung einer Situation verändern kann, was bis zum Auflösen einer laufenden Interaktion führen kann. Dieser Blick auf Aktivitäten in Interaktionen ist nur möglich, wenn sich Forschende von der häufig sprachzentrierten Perspektive in der Interaktionsforschung lösen und sprachfreie Aktivitäten und solche mit verbaler Beteiligung gleichberechtigt nebeneinander betrachten. Ein Vorschlag für diese Perspektive findet sich in den Typisierungen von Aktivitäten als Ensemble- und Einzelaktivitäten. Die vorliegende Arbeit trägt auf diese Weise dazu bei, den Begriff Aktivität als notorische „fuzzy category“ (Levinson 1992: 69) der Konversationsanalyse in ihrer Konzeption zu festigen und für Folgestudien nutzbar zu machen. Die daraus resultierende, konsequent multimodal-kommunikationswissenschaftliche Betrachtungsweise macht es im Rahmen der konversationsanalytischen Methodologie möglich, auch Koordinationsprozesse von und mit Aktivitäten ohne verbaler Beteiligungsweisen zu analysieren, indem das zentrale Prinzip des next turn/action proof procedure um die Prozesse des Monitorings und Displayings erweitert wird. Damit trägt die vorliegende Arbeit zum bislang noch recht überschaubaren Feld der Multiaktivitätsforschung bei und lenkt den Blick auf jene größeren interaktionalen Kontexte der Ebene einer supra-sequenziellen Kohärenz (Robinson 2013: 258), die im Rahmen konversationsanalytischer Studien oft - bedingt durch die Fokussierung auf sequentielle Phänomene im Bereich der Handlungen oder Praktiken - ausgeklammert werden. 
